\title{
Seismic Behaviour of Bolted and Bonded Point Fixed Laminated Glass Panels
}

\author{
Eliana Inca $^{1}$, Chiara Bedon ${ }^{2}$, Sandra Jordão ${ }^{1}$, and Carlos Rebelo ${ }^{1}$ \\ ${ }^{1}$ University of Coimbra, Institute for Sustainability and Innovation in Structural Engineering (ISISE), \\ Department of Civil Engineering, 3030 - 790 Coimbra, Portugal \\ ${ }^{2}$ Department of Engineering and Architecture, University of Trieste, Piazzale Europa 1, 34127 \\ Trieste, Italy
}

\begin{abstract}
Laminated glass panels with point fixing restraints are widely used in buildings. Although significant progress has been reached within its production and installation, a lack of standardization, and particularly, their actual load-bearing capacity and performance under seismic events is not well addressed, and is largely affected by the connection detailing and typology. The present research study explores the seismic performance of single laminated glass panels with various point fixing solutions. The characterization involves experimental set ups and numerical models.
\end{abstract}

\section{Introduction}

Glass facade industry has experienced an exponential growth since its first applications in late 60's [1]. Point fixed glass systems and particularly bonded connections, have been used to fulfil the requirements of transparency and challenging structures, and a variety of applications have been already constructed such as stairs, canopies, roofs, to complex facade systems of tall buildings and open spaces, art galleries, museums, among others (Figure 1 and [2]). As opposed to its growing use in construction and the great technological developments reached in production and installation, structural designers still encounter a void in regard to standardisation and recommendations for these structures [3]. Thus, several glass systems have been designed with piecewise procedures, including full scale testing and even complex numerical modelling, to support pioneer design [4]. Particularly, the overall response of point fixed glass facade systems under seismic actions is not yet fully studied. Further, specific technical regulations are still missing. Some structural design standards, for example, would classify glass facades as merely secondary elements, disregarding the lateral load transfer from the main building, and therefore assuming no response from different facade systems to certain dynamic loads. This is a fundamental topic, given that although glass is surprisingly deformable and able to accommodate significant displacements, it is critically susceptible to harsh contact, which may occur during a seismic event at the panel contour (in supported panels), or at the connectors (in point supported facades). The final aim of this study is hence to develop design guidelines and retrofitting reports for pointed fixed glass facade systems when subjected to seismic loads.

\footnotetext{
${ }^{*}$ Corresponding author: chiara.bedon@dia.units.it
} 


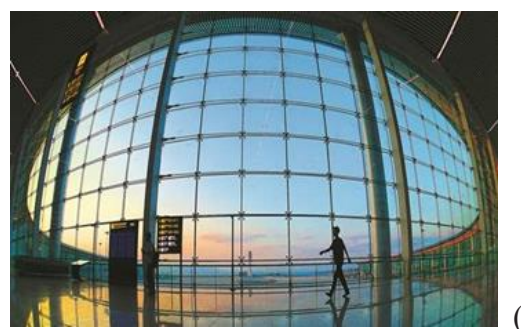

(a)

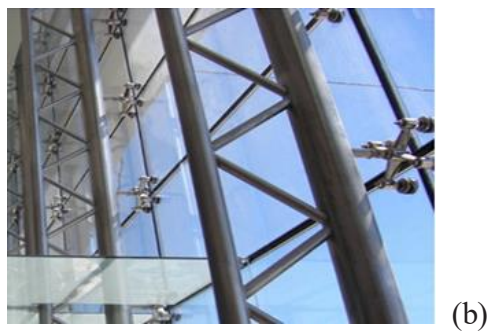

Fig. 1. Examples of facades: (a) cabled curtain wall at Chongqing Airport T3, China (Architectural concept: ADPI and Chengdu Institute of Architecture, 2017); (b) pointed fixed system.

\section{Reported Damage to Glass Facades in Past Earthquakes}

After the San Fernando earthquake in 1971 (California), where several buildings were reported with glass damage [5], it became clear that damage of non-structural components can be very costly but also can pose real threat to life safety. Several strong earthquakes reported since 1994's Northridge event have shown damage on medium high buildings (812 stories) mostly due to structural irregularities. Important non-structural damage was observed on in-fill walls (with masonry facades severely damaged), but window glass falling was the second most serious damage well documented [6-12], see Figure 2.

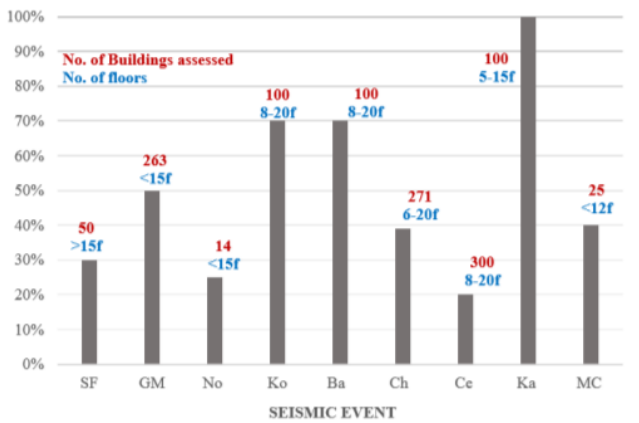

\begin{tabular}{|c|c|c|c|}
\cline { 2 - 4 } \multicolumn{1}{c|}{} & Location & Date & Mw \\
\hline SF & San Fermando, Califorria, USA & 9-Feb-71 & 6.5 \\
\hline GM & Guerrero-Michoacan, Mexico & $19-S e p-85$ & 8 \\
\hline No & Northridge, California, USA & $17-E n-1994$ & 6.7 \\
\hline Ko & Kobe, Japan (100Blds) & 17-Ian-95 & 6.9 \\
\hline Ba & Bam, Iran & 26-Dec-2003 & 6.6 \\
\hline Ch & Crristchurch, New Zeland & 22-Feb-2011 & 6.3 \\
\hline Ce & Central, Italy & 30-Ago-2016 & 6.5 \\
\hline Ka & Kaikōura, Japan & 13-Nov-2016 & 7.8 \\
\hline MC & Mexico City, Mexico & 19-Sep-2017 & 7.1 \\
\hline
\end{tabular}

Fig. 2. Percentage of facade damage in buildings per major seismic events.

An important correlation between the inter-storey drift and the glass damage was reported on several post-earthquake damage assessments [8]. Flexible glass facade systems enclosed by metal curtain walls and mullions presented less damage in past seismic events [12], mainly due to the high deformability of silicon allowing for a better accommodation of drift demand during ground shaking. Furthermore, glass damage consistently increases with larger window areas and irregular plan configurations [13].

\subsection{Code Provisions for In-Plane Drift Demands}

For the design of buildings under seismic events, most of the current design code provisions include specifications for Inter-story Drift Ratios (IDRs) limits under lateral loads. Generally, those limits are specified according to the building typology and features. No straight specifications indeed exist for glass facades. For both primary or secondary glass elements, rigid resistance and deformation requirements are generally recommended [4]. Current practice specifies to develop experimental set-ups for non-typical configurations. Regarding curtain walls, a standard test protocol is provided in (AAMA 501.6 [14]) for the determination of the seismic drift causing glass fallout. 


\section{Bolted and Bonded Point Fixings}

\subsection{Selected Fixings}

The present project involves experimental, numerical and analytical verifications that are conducted first on single point fixed laminated glass (LG) panels to address the response under dynamic and/or cyclic loads. Different types of connections re used, such as embedded, countersunk and very promising bonded point fixations are considered. Among others, enhanced countersunk fixings (Figure 3(a)) allow to transfer out of plane and in plane loads directly through the bolt and glass interface. Alternatives are represented by embedded bolts (Figure 3(b)), that reduce the concentration of stresses around the boreholes, and adhesive connections (Figure 3(c)), that avoid the throwbacks of drilling the glass, because the glass is directly bonded to the metal connector.

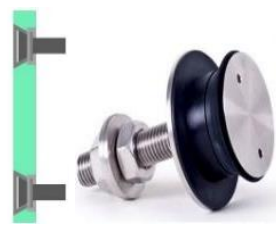

(a)

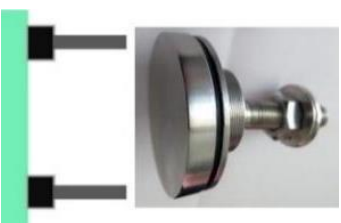

(b)

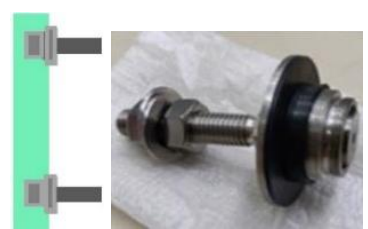

(c)

Fig. 3. Different types of point fixed connectors for glass panels: (a) countersunk; (b) embedded bolts and (c) bonded articulated stainless steel bolts.

At the first stage of this study, LG panels are subjected to uniform distributed load, simulating wind pressure (Figure 4). Later on, the panels will be subjected to cyclic load and finally the overall behaviour of a full-scale facade systems will be assessed under lateral loads.
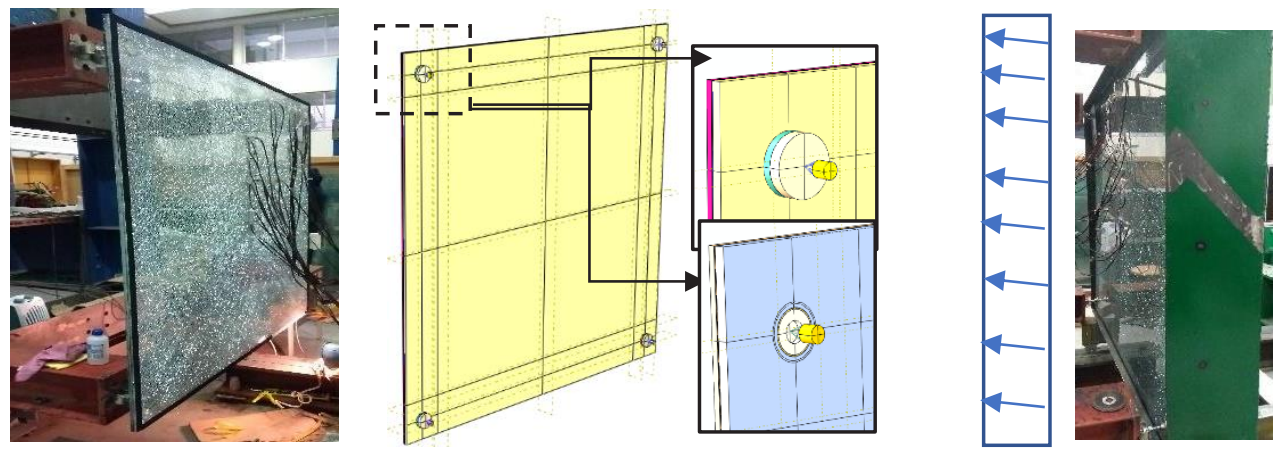

Fig. 4. Set-up for a single LG panel with point fixings at the corners and subjected to uniform distributed load: experimental analysis and numerical modelling.

\subsection{Cyclic Load Pattern}

The determination of the amount of lateral load on a facade and/or a single panel is a very sensitive topic, since there are several parameters involved. The lack of standard load protocols for these systems on one side, and the variability of degree for the load transfer from the primary building to the facade makes the issue complex. Major influencing parameters are secondary supports, connections on top and bottom of the facade, structural system, seismic performance of the building, and most importantly the local seismicity.

The influence of the primary structure for the facade systems is the main interest of the current research. More in detail, the study of a mid-heigh building, subjected to seismic load and its design according to current standards for buildings (as in Figure 5) is the basis to 
obtain the input parameters in terms of maximum lateral displacements for both the perpendicular directions.
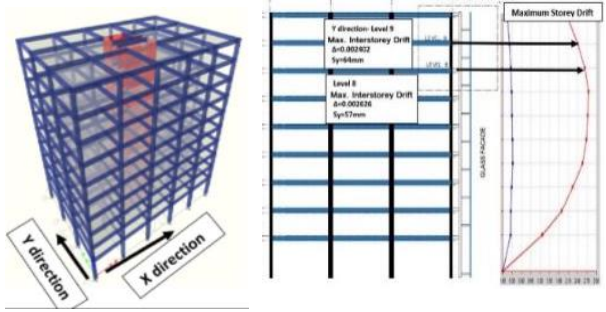

(a)

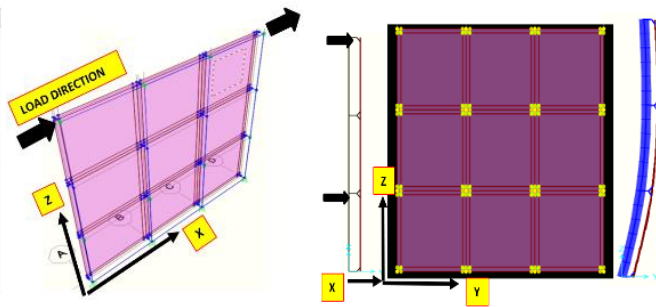

(b) (c)

Fig. 5. Transfer of lateral loads form building to a section of a glass facade system: (a) assessment of lateral displacements for a concrete building; and detail of facade under (b) in-plane lateral or (c) out-of-plane displacements.

\subsection{Results}

Square LG panels $(1500 \times 1500 \mathrm{~mm}, 6 \mathrm{~mm}+10 \mathrm{~mm}$ the thickness $)$ with fixations as in Figure 3 (placed $80 \mathrm{~mm}$ from each corner) were experimentally investigated. In addition to different fixings, LG sections were tested with both PVB and EVA interlayers, and subjected to uniform distributed loads (up to $8 \mathrm{kPa}$ ). Numerical models for the same LG panels are currently investigated using ABAQUS (Figure 6), based on full solid elements (C3D8R). Linear elastic material properties are assigned to glass, interlayers and steel, while different hyperplastic laws are used for the silicone bonded solution. The preliminary results show high variability on the properties of interlayer for different load rates, that might be attributed to the laboratory temperature conditions on storage for the laminates. Further, the sensitivity of different hyperelastic parameters is addressed towards experimental measurements.

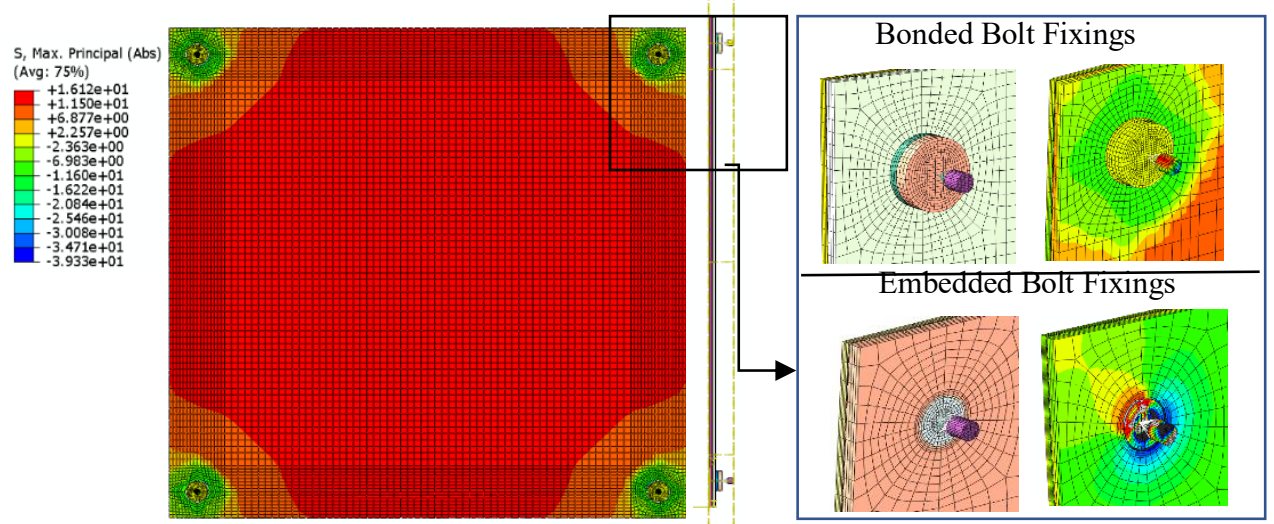

Fig. 6. Numerical analysis of LG panel with two types of fixations. In evidence, the stress peaks at the restraints.

\section{Conclusions}

Besides largely used in buildings, glass structures and facades still represent a challenging task for designers. In most of the cases, no specific design recommendations are given by regulations and codes, or even testing protocols, with major issues for designers. The current study explores the seismic performance of laminated glass panels with different types of point fixing restraints. 


\section{Acknowledgments}

The Portuguese Foundation for Sciences and Technology (Fundação da Ciência e Tecnologia) under contract grant POCI-01-0145-FEDER-032539 (GF-Seismic | Structural Glass Facades Subjected to Seismic Load) is gratefully acknowledged. The EU-COST Action CA18120 (CERTBOND, https://certbond.eu/) is also acknowledged for providing financial support to the first author (STSM visitor at University of Trieste, Italy). Finally, the EDES2021 Conference presentation is supported by the ongoing "Multiwall" FRA-2020 project at University of Trieste.

\section{References}

1. J. Vitkala, https://www.gii.fi/@Bin/199723/trendsgpd2017text_eng1_vr4.pdf, Glass Performance Days (2017)

2. C. Bedon, M. Santarsiero, Advanced Engineering Materials, 20, 5: 1700815 (2018), doi: 10.1002/adem.201700815

3. C. Bedon, X. Zhang, F. Santos, D. Honfi, M. Kozlowski, M. Arrigoni, L. Figuli, D. Lange, Construction and Building Materials, 63, 28: 921-937 (2018), doi: 10.1016/j.conbuildmat.2017.12.153

4. C. Bedon, C. Amadio, S. Noé, Safety, 5, 4: 80 (2019), doi: 10.3390/safety5040080

5. P.C. Jennings, Earthquake Spectra, 3, 1 (1997), doi: 10.1193/1.1585930

6. D. Evans, F.J.L. Ramirez, Reducing Earthquake Hazards: Lessons Learned from the 1985 Mexico Earthquake, pp. 234-236, Publ by Earthquake Engineering Research Inst, (1989)

7. S.M.Q. Roeslin, Q.T.M. Ma, H.J. García, Frontiers in Built Environment, 4: 1-18 (2018), doi: 10.3389/fbuil.2018.00072

8. A. Baird, A. Palermo, S. Pampanin, Bulletin of the New Zealand Society for earthquake engineering, 44, 4: 368-376 (2011), doi: 10.5459/bnzsee.44.4.368-376

9. A. Baird, H. Ferner, Bulletin of the New Zealand Society for Earthquake Engineering, 50, 2: 187-193 (2017), doi: 10.5459/bnzsee.50.2.187-193

10. M. Hosseini, Earthquake Spectra, 21, 1: 439-453 (2005), doi: 10.1193/1.2098829

11. R.A. Behr, Architectural glass to resist seismic and extreme climatic events, Elsevier, 272 pages, (2009)

12. D. Perrone, P.M. Calvi, R. Nascimbene, E.C. Fischer, G. Magliulo, Bulletin of Earthquake Engineering, 17, 10: 5655-5677 (2019), doi: 10.1007/s10518-018-0361-5

13. H. Sucuoglu, C.G. Vallabhan, Engineering structures, 19, 8: 685-694 (1997)

14. AAMA 501.6:2018, Recommended Dynamic Test Method for Determining the Seismic Drift Causing Glass Fallout from Window Wall, Curtain Wall and Storefront Systems 